\title{
Plasmon-directed polymerization: Regulating polymer growth with light
}

\author{
Yunxia Wang ${ }^{1}$, Shuangshuang Wang ${ }^{1}$, Shunping Zhang ${ }^{1}$, Oren A. Scherman ${ }^{3}$, Jeremy J. Baumberg ${ }^{2}$, \\ Tao Ding ${ }^{1,2}(\varangle)$, and Hongxing $\mathrm{Xu}^{1}$ \\ ' Key Laboratory of Artificial Micro- and Nano-structures of Ministry of Education of China, School of Physics and Technology, Wuhan \\ University, Wuhan 430072, China \\ ${ }^{2}$ Nanophotonics Centre, Cavendish Laboratory, University of Cambridge, Cambridge, CB3 OHE, UK \\ ${ }^{3}$ Department of Chemistry, University of Cambridge, Lensfield Road, Cambridge, CB2 1EW, UK
}

Received: 21 June 2018

Revised: 24 July 2018

Accepted: 28 July 2018

(C) The author(s) 2018. This article is published with open access at link.Springer.com

\section{KEYWORDS}

hot electrons, plasmons, polarization, selective growth

\begin{abstract}
Plasmon-induced polymerization can facilitate the site-selectivity and orientation of polymer growth, which enriches the toolbox of polymerization and nanofabrication. Here, we demonstrate plasmon-induced polymerization, accomplished by low-power laser excitation of gold nanoparticles (NPs). We selectively control the growth of polymers around single plasmonic NPs while monitoring the polymerization using dark field spectroscopy and subsequent scanning electron microscopy. This plasmon-induced polymerization, generated by hot electron initiation, not only precisely controls the thickness and composition of the polymer coatings but also regulates the location and orientation of the growth, which are strongly influenced by the laser polarization and near-field distribution around the plasmonic NPs. A saturation increase in the polymer thickness provides a strong support for our mechanism. This facile approach of nanoscale polymerization directed by light not only provides new opportunities in nanosynthesis and nanofabrication for functional devices, but also opens many routes for polymer physics and chemistry at the nanoscale level.
\end{abstract}

\section{Introduction}

Plasmon-induced chemistry via hot carriers has attracted great interest as it opens a new paradigm of nanochemistry for self-assembly [1], plasmonics [2], photodetection [3], photocatalysis [4, 5] and photovoltaics [6-8]. It is generally believed that plasmon- excited hot carriers trigger a series of redox chemistries at the surface of metals or semiconductors via charge transfer [9]. Such injected hot electrons can also be utilized to induce surface polymerization, which then shifts the tuning of the plasmonic resonances with a feedback that can be self-limiting [2].

This hot-electron polymerization is quite different

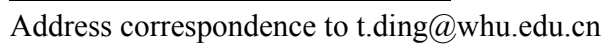


from plasmon-assisted two-photon polymerization, which usually works for photosensitive materials such as SU-8 [10-14]. Instead, polymerization via hot electrons appears to initiate most types of radical polymerization reactions, which significantly broadens its application. The advantage of using plasmoninduced hot electrons, compared to conventional surface-initiated radical polymerization, is the precise location and polarization dependence it gives, which strongly improves the precision for fine-tuning nanostructures. This bridges a distinct gap for the fabrication of miniaturized polymer optoelectronic devices, where excellent site-selectivity, and remote controllability and directionality are demanded.

The rationale here is to control the polymerization location and direction by irradiating with a linearly polarized laser beam at $\lambda=640 \mathrm{~nm}$ (Fig. 1(a)). Performing the laser illumination in an optical microscope allows simultaneous dark-field spectroscopy to monitor the polymerization process in real-time. Plasmon-induced polymerization is observed here for different types of monomers, even without the presence of initiators, but is found to terminate at a critical thickness. This strongly suggests that the hot electrons from $\mathrm{Au}$ can initiate the radical polymerization of only the few monomers positioned close to the metal surface (Scheme 1) [2]. The time- and power-dependent growth provides a facile means to control the thickness of the polymer coating. The polymer growth shows polarization dependence with an aspect ratio of $\sim 1.65$, due to localization of the hot $\mathrm{e}^{-}$injection [15]. The growth position can also be controlled by the position of the hotspots, where the efficiency of hot $\mathrm{e}^{-}$injection is maximized. Although point-by-point laser writing is not suitable for the mass production of polymers, this method opens new opportunities for polymer-based nanodevice fabrication with precise local selectivity and directionality, which traditional mix-and-stir chemistry (including surfaceinitiated polymerization) cannot support.

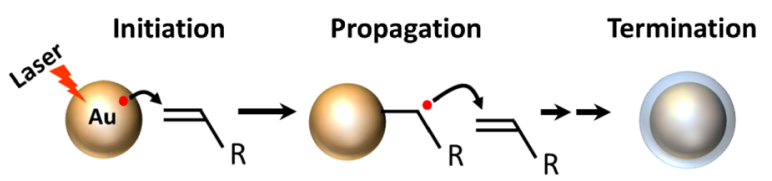

Scheme 1 Mechanism of hot-electron-initiated surface polymerization.

\section{Results and discussion}

\subsection{Scheme for polymerizing by light}

Our aim in this work is to avoid complications from oxides by using only $\mathrm{Au}$ and to prevent Schottky barrier effects by avoiding semiconductor layers such as $\mathrm{ZnO}$ or $\mathrm{TiO}_{2}$, thus escaping any spurious photochemistry. We also try to avoid further complexity through the use of neat-monomer liquids, such as divinylbenzene (DVB), styrene (St), or methyl methacrylate (MMA), while performing laser irradiation (Fig. 1(a)). We characterized the optical response of single Au nanoparticles via dark-field microscopy, comparing their scattering cross-sections before and after irradiation (inset Fig. 1(b)). The laser irradiation induced $\mathrm{a} \sim 30 \mathrm{~nm}$ redshift of the plasmonic resonance due to the increased refractive index of the polymer coatings, which is supported by the simulations (Fig. 1(c)). The PDVB coating can be clearly visualized in the scanning electron microscopy (SEM) images (Fig. 1(d)), confirming successful polymer growth around the Au NP.

Such a polymerization process is not likely due to chemical initiation, as no initiators were used, and the temperature rise was less than $30^{\circ} \mathrm{C}$ with a laser wavelength at the $2 \mathrm{~mW}$ laser power here (see section 2.2) [16], which is much less than required for effective auto-initiation $\left(>80^{\circ} \mathrm{C}\right.$ for most monomers, and which can only be seen at higher powers, see section 2.2) [17]. We also applied plasma treatment to remove the organic ligands coating the Au NP that might potentially cause initiation, but still polymer growth was observed (Fig. S1 in the Electronic Supplementary Material (ESM)). Direct laser-induced photo-initiation can also be excluded since no polymer growth was observed on the flat $\mathrm{SiO}_{2} / \mathrm{Si}$ surfaces, even at high powers $(>10 \mathrm{~mW})$. While near-field plasmon enhancements can increase local light intensities to initiate radical polymerization [18-20], photon energies of $1.95 \mathrm{eV}$ are not sufficient to directly produce free radicals from the monomers. One of the indirect proofs of hot $\mathrm{e}^{-}$initiation is that no polymer growth was observed for $\mathrm{Al}_{2} \mathrm{O}_{3}$ coated Au NPs ( $2 \mathrm{~mW}$, Fig. S2 in the ESM) as the insulating layer blocks the hot $\mathrm{e}^{-}$from injecting into the monomers. At high power 
(a)
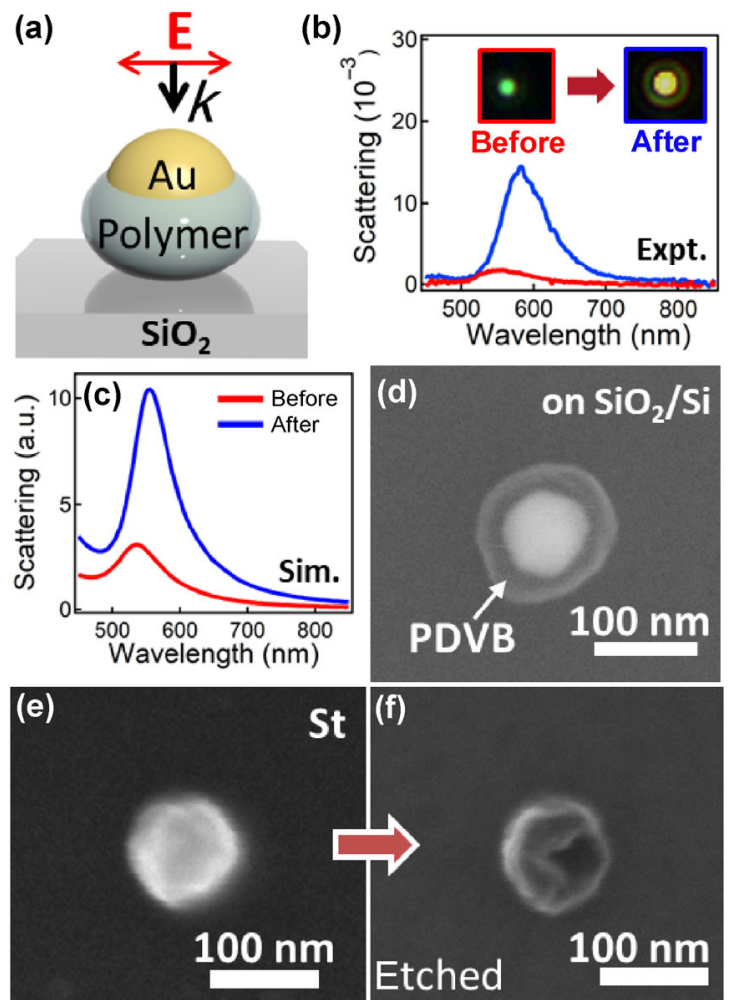

St (f)

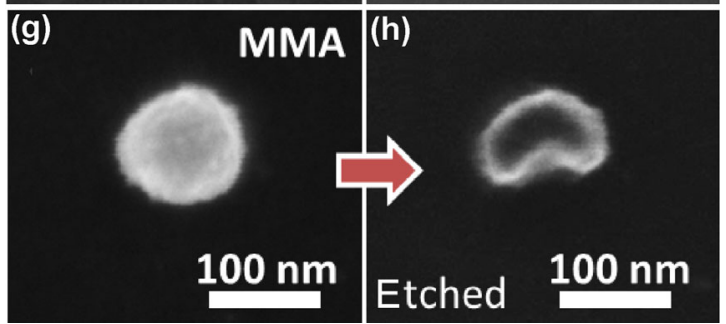

Figure 1 Laser-induced polymerization. (a) Pump laser $(\lambda=$ $640 \mathrm{~nm}, 2 \mathrm{~mW}, 60 \mathrm{~s}$ ) excites $\mathrm{Au} \mathrm{NPs}$ on $\mathrm{SiO}_{2} / \mathrm{Si}$ wafer inducing polymer growth around the NP. (b) Scattering spectra (referenced to white diffuser) of $\mathrm{Au} \mathrm{NP}$ before/after laser irradiation (insets show corresponding dark field images). (c) Simulated scattering spectra of $\mathrm{Au}(80 \mathrm{~nm})$ and $\mathrm{Au} @$ PDVB on $\mathrm{SiO}_{2} / \mathrm{Si}$ substrate. (d)-(h) SEM images of irradiated Au NP in bulk monomer of (d) DVB, (e) St, (g) MMA, and (f) and (h) after etching of Au core with Lugol solution $\left(\mathrm{KI} / \mathrm{I}_{2}\right)$.

$(8 \mathrm{~mW})$, a significant growth of PDVB was observed but this is due to auto-initiation (see section 2.2).

Other monomers, including styrene (St, Fig. 1(e)) and methyl methacrylate (MMA, Fig. 1(g)), were also found to polymerize around the Au NPs with laser irradiation, which is verified by the leftover buckled polymer shells after the Au cores were etched out (Figs. 1(f) and 1(h)). The polymer shell was significantly thinner in these cases compared to PDVB (Fig. 1(d)). We suggest that this is likely due to their different termination kinetics, since hot electrons will generate radicals in a fraction of monomers close to the surface at similar rates. Termination through radical collisions, either by radical-radical combination or by disproportionation, is dependent on the monomer chemistry as well as the local radical concentration (Scheme 1). The thicker shells formed by PDVB around these Au NPs may be due to the reduced chain termination rate arising when PDVB crosslinks, which sterically reduces the chances of two radical chain ends meeting.

\subsection{Nonlinear optical power dependence}

To further distinguish hot $\mathrm{e}^{-}$induced polymerization from optical heating induced polymerization, we investigated the influence of laser power (Fig. 2) on the thickness of the polymer shells around each $\mathrm{Au}$ NP. Although hot electron generation is not expected to require a power threshold, the energy of the hot electrons will change their escape probability, introducing a dependence on laser wavelength and intensity. As a result, we found that the polymer coatings are only seen in SEM when the laser irradiation power exceeds $\sim 0.5 \mathrm{~mW}$ (Fig. 2(b)), while the polymer growth is then limited by the number of monomers that are present on the metal surface, thus giving a constant $25 \mathrm{~nm}$ thickness (Figs. 2(c) and 2(d)). At high powers $(>6 \mathrm{~mW})$, optical heating triggers the auto-initiation of polymerization throughout a much larger volume away from the Au NP surface (Fig. 2(e)). Simulations with COMSOL (Fig. S3 in the ESM) confirm such heating effects are substantial enough to give polymerization when the surface temperature of $\mathrm{Au}$ NPs exceeds $400 \mathrm{~K}$ (laser power $8 \mathrm{~mW}$, Fig. 2(g)). However, for low power irradiation $(2 \mathrm{~mW})$, the temperature rise around each $\mathrm{Au} \mathrm{NP}$ is only $~ 27 \mathrm{~K}$ (Fig. 2(f)), and polymerization at this temperature $\left(47^{\circ} \mathrm{C}\right)$ is not due to optical heating but rather hot $\mathrm{e}^{-}$ effects. This nonlinear power dependence of polymer growth (Fig. 2(h)) clearly suggests two different modes of initiation: at powers between 0.5 and $4 \mathrm{~mW}$, hot $\mathrm{e}^{-}$initiation is dominant; at irradiation powers above $6 \mathrm{~mW}$, polymerization is mainly triggered by auto-initiation, while in-between, both hot $\mathrm{e}^{-}$and auto-initiation may coexist (Fig. 2(d)). 


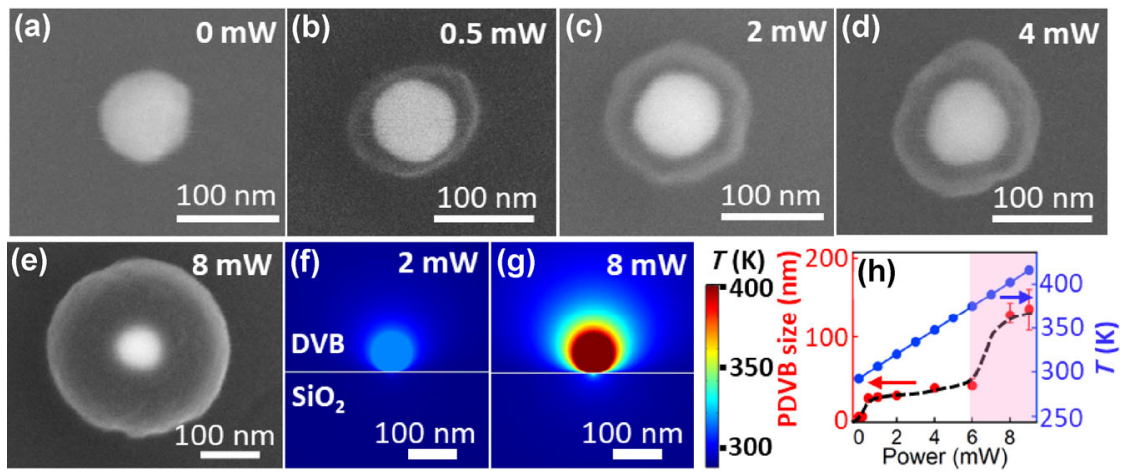

Figure 2 Power dependence of laser-induced polymerization around Au NPs in DVB monomer. Representative SEM images of irradiated $\mathrm{Au}$ NPs at different average powers: (a) 0 , (b) 0.5 , (c) 2, (d) 4 , and (e) $8 \mathrm{~mW}$ for $60 \mathrm{~s}$. Simulations (COMSOL) of temperature distribution around irradiated Au NP at different laser powers of (f) 2 and (g) $8 \mathrm{~mW}$. (h) Change of shell thickness (experiment) and surface temperature (from simulation) of Au NPs with irradiation power. Each condition is repeated over 4-5 particles to confirm their validity.

\subsection{Time-dependent growth}

We also investigated the influence of irradiation time (Fig. 3) on the shell thickness of PDVB. Anisotropic growth was observed around the Au NP in the first $20 \mathrm{~s}$ (Figs. 3(a)-3(d)), due to the polarization of the laser (see section 2.4). After $30 \mathrm{~s}$, such anisotropy disappeared as the polymer shell tried to reduce its surface-to-volume ratio and a uniform coating was observed (Figs. 3(d)-3(g)). The polymer shell thickness increased dramatically in the first $30 \mathrm{~s}$ of irradiation, then slightly decreased (Fig. 3(h)). The volume shrinkage is probably due to the crosslinking of the PDVB matrix, which also leads to the increase in the refractive index of the coatings. As a result, the plasmon peaks were slightly redshifted, even when the size of the coatings decreased (Fig. S4 in the ESM).

\subsection{Polarization dependence}

The growth of PDVB at the early stage (Figs. 3(c) and 3(d)) or with lower power (Fig. 2(b)) shows anisotropic features, which eventually merge into a uniform coating (Fig. 3(e)). The preferential growth at the opposite ends of the Au nanospheres arises from the plasmon mode which is excited by polarized light along the same direction [11, 12], thus generating hot $\mathrm{e}^{-}$only in these directions [15]. To further verify this, we rotated the direction of the linearly polarized laser and observed how the orientation of polymer growth changed (Fig. 4). Clearly, the polymer growth is preferential along the laser polarization with an
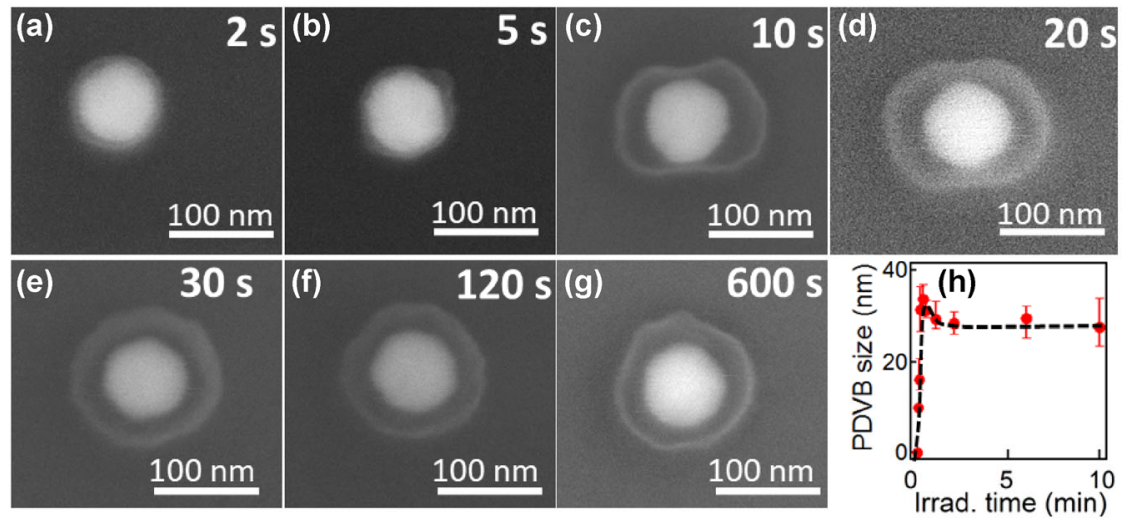

Figure 3 Increase in times of laser-induced polymerization around Au NPs in DVB monomer. Representative SEM images (a)-(g), durations of $2,5,10,20,30,120$, and $600 \mathrm{~s}$ at $2 \mathrm{~mW}$, with (h), mean shell thickness vs. irradiation time. Each condition is repeated over 4-5 particles to confirm their validity. 


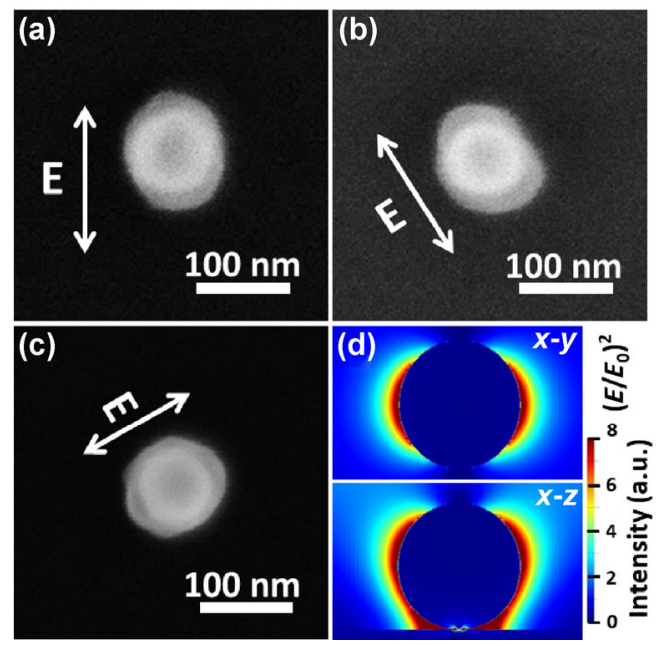

Figure 4 Irradiating Au NPs/DVB on Si substrates with different laser polarization directions $(0.5 \mathrm{~mW}, 60 \mathrm{~s})$. (a)-(c) SEM images of Au NPs with different polarization orientations. (d) FDTD simulations of the near-field profiles of Au NPs on Si substrate with surrounding medium of DVB $(n=1.59)$.

overall aspect ratio of $1.2 \pm 0.5$ (Figs. 4(a)-4(c)) (with a polymer coating aspect ratio of $1.65 \pm 0.5$ ). The near-field simulations (Fig. 4(d)) reveal the polarizationdependent profiles, indicating hot $\mathrm{e}^{-}$injection is maximized along the polarization direction, and thus polymer protrusion occurs preferentially in the alignment of polarization. This directional growth set by the light polarization significantly advances the controllability of polymer growth for functional nanodevices.

\subsection{Local selective polymer growth with plasmons}

As hot $\mathrm{e}^{-}$injection efficiency is proportional to the near-field intensity, polymerization selectively happens at the location where the field intensity is the strongest. Therefore, plasmonic hotspots that confine the electric field to the nanoscale can be used to initiate polymerization with sizes far below the diffraction limit. In this way, we can control the location of polymer growth with sub-wavelength resolution (Fig. 5). As the excitation of plasmon modes is highly dependent on the incident polarization, polymer growth can then be selectively driven at different locations of the plasmonic NP. For instance, Au bipyramid nanoparticles (BPNs) have stronger longitudinal modes and weaker transverse modes (insets of Figs. 5(a) and 5(b)), so that excitation along the two different axes leads to selective growth either at their tips or at their sides (Figs. 5(a) and 5(b)). As the field intensity is 50 times stronger at the tips than at the sides, the size of PDVB growth is much larger at the tips. Note that the shapes and sizes of polymers do not exactly follow the shape of the near-field distribution, which further suggests it is plasmons initiating the polymer growth rather than acting as a local source for light-induced polymerization. We can also vary the irradiation power to control the coating thus changing from small bulbs at the tips, to full encapsulation of the whole Au BPNs (Fig. S5 in the ESM). The same principle also applies for Au dimers (Fig. 5(c)) and Au prisms (Fig. 5(d)), where the growth favorably happens at the location where intense electric fields are selectively excited by the polarized light (insets of Figs. 5(c) and 5(d)). As the field enhancement in the nanogap of an Au dimer is 3 orders of magnitude higher than normal open plasmonic nanostructures, the polymer growth in the nanogap region extends to a much larger area (Fig. 5(c)), suggesting the near field enhancement may accelerate the rate of hot $\mathrm{e}^{-}$injection. Selective polymer growth can also be accomplished on lithographically-fabricated nanostructures (Fig. S6 in the ESM) [21,22], which provides a useful technique towards functional nanophotonic and nanoelectronic devices.
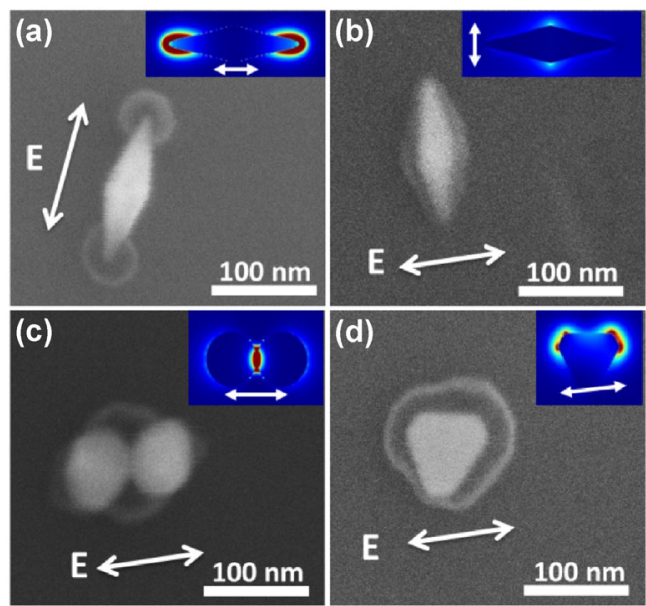

Figure 5 Plasmon-induced polymerization with different shaped Au NPs forming the plasmonic resonator. (a) and (b) Au BPNs, irradiation condition: $0.75 \mathrm{~mW}, 30 \mathrm{~s}$; (c) Au dimers, irradiation condition: $0.8 \mathrm{~mW}, 30 \mathrm{~s}$; (d) Au prisms, irradiation condition: $0.5 \mathrm{~mW}, 30 \mathrm{~s}$. Insets are the FDTD simulations of their corresponding near-field profiles, color scales are in arbitrary units which are not normalized. 


\section{Conclusions}

Plasmon-induced polymerization has been shown to provide unique advantages for polymer growth with nanoscale precision. The thickness of the polymer coating produced varies from sub- $10 \mathrm{~nm}$ up to $150 \mathrm{~nm}$ depending on the duration and power of the irradiation, with control at the $1 \mathrm{~nm}$ scale using simultaneous optical measurements. From the nonlinear power-dependent growth and the general consistency for different monomers, we confirmed such polymerization is due to the injection of hot electrons generated by the plasmons. These hot electrons have energies of several $\mathrm{eV}$, much larger than typical thermal energies ( $30 \mathrm{meV}$ ) but much less than high-energy gamma-ray initiators (keV), and thus generate highly-local but initiator-free radical polymerization from metal surfaces [23]. Furthermore, the location and orientation of polymer growth can also be controlled with optical polarization due to the near-field distribution of the plasmonic components which directs hot $\mathrm{e}^{-}$ejection. This facile polymerization, induced simply by laser irradiation, provides a unique control over polymer growth with different sizes, compositions, positions, and orientations, significantly enriching the versatility of current polymerization techniques. Although this technique is not scalable for polymer production, it opens investigations of many questions and applications, such as in co-polymerization chemistry (Fig. S7 in the ESM) and ultrathin dielectric coatings for capacitors and transistors.

\section{Method}

Au NPs (80 nm, obtained from BBI) and Au bipyramid NPs (obtained from Nanoseedz) were drop-cast on oxygen-plasma-cleaned $\mathrm{SiO}_{2}(400 \mathrm{~nm})$ /silicon wafers. Monomers (with inhibitors removed using $10 \mathrm{wt} \%$ $\mathrm{NaOH}$ solution) were drop-cast onto the samples, which were then covered with a coverslip for microscope observation (Scheme S1 in the ESM). A $640 \mathrm{~nm}$ continuous wave (CW) laser (Coherent Cube) was free-space coupled to the microscope, which was then focused down onto the particles through a 100× objective (Olympus, NA=0.8). The irradiation occurred with the presence of a monomer liquid, and the duration and laser power were varied to investigate polymerization conditions. At least 5 particles were irradiated for each condition to assure the validity of the obtained data. The scattering spectra were taken confocally through a $50 \mu \mathrm{m}$ optical fiber coupled to a spectrometer (QEPro, Ocean Optics) before and after irradiation. To change the polarization of laser incidence, a linear polarizer and a half-wave plate were placed and rotated before the objective lens. The irradiated particles were tracked and characterized with scanning electron microscopy (Zeiss). The temperature distribution around the Au NPs was simulated with COMSOL Multiphysics 5.3 (see ESM for details). The optical near-field and scattering cross-section of the $\mathrm{Au}$ NPs were calculated with Finite Difference Time Domain (FDTD) Solutions (Lumerical). Broadband plane waves (450-950 nm) were normally incident, with the refractive index of $\mathrm{Au}$ taken from Johnson and Christy [24], and meshing sizes of $0.8 \mathrm{~nm}$. The boundary conditions were set to be symmetric in the $y$ direction and asymmetric along the $x$ direction (incidence plane).

\section{Acknowledgements}

This research is supported by the Fundamental Research Funds for the Central Universities (Nos. 2042018kf0038 and 2042018kf0254), the National Natural Science Foundation of China (NSFC) (Nos. 21703160 and 11674256), Major State Basic Research Development Program (No. 2015CB932400) and UK EPSRC EP/ G060649/1 and EP/L027151/1. We thank Jibo Tang for the preparation of the bowtie-like electrodes.

Electronic Supplementary Material: Supplementary material (thermal calculations with COMSOL; experimental setup scheme; SEM image of PDVB coating on $\mathrm{Au}$ NP which is cleaned with oxygen plasma; SEM images of $\mathrm{Al}_{2} \mathrm{O}_{3}$ protected Au NPs after irradiation; scattering spectra of $\mathrm{Au}$ NPs before and after laser irradiation for different durations; SEM images of PDVB coatings on Au BPNs with increasing irradiation power; polymerization in lithographic plasmonic nanostructure, and plasmon induced co-polymerization) is available in the online version of this article at https://doi.org/10.1007/s12274-018-2163-0. The raw data 
of the figures in this paper can be found at https:// doi.org/10.17863/CAM.26349.

Open Access: This article is distributed under the terms of the Creative Commons Attribution 4.0 International License (http://creativecommons.org/licenses/ by/4.0/), which permits unrestricted use, distribution, and reproduction in any medium, provided you give appropriate credit to the original author(s) and the source, provide a link to the Creative Commons license, and indicate if changes were made.

\section{References}

[1] Cortés, E.; Xie, W.; Cambiasso, J.; Jermyn, A. S.; Sundararaman, R.; Narang, P.; Schlücker, S.; Maier, S. A. Plasmonic hot electron transport drives nano-localized chemistry. Nat. Commun. 2017, 8, 14880.

[2] Ding, T.; Mertens, J.; Lombardi, A.; Scherman, O. A.; Baumberg, J. J. Light-directed tuning of plasmon resonances via plasmon-induced polymerization using hot electrons. ACS Photonics 2017, 4, 1453-1458.

[3] Knight, M. W.; Sobhani, H.; Nordlander, P.; Halas, N. J. Photodetection with active optical antennas. Science 2011, 332, 702-704.

[4] Kim, M.; Lin, M. H.; Son, J.; Xu, H. X.; Nam, J. M. Hotelectron-mediated photochemical reactions: Principles, recent advances, and challenges. Adv. Opt. Mater. 2017, 5, 1700004.

[5] Sun, M. T.; Xu, H. X. A novel application of plasmonics: Plasmon-driven surface-catalyzed reactions. Small 2012, 8 , 2777-2786.

[6] Clavero, C. Plasmon-induced hot-electron generation at nanoparticle/metal-oxide interfaces for photovoltaic and photocatalytic devices. Nat. Photonics 2014, 8, 95-103.

[7] Mukherjee, S.; Libisch, F.; Large, N.; Neumann, O.; Brown, L. V.; Cheng, J.; Lassiter, J. B.; Carter, E. A.; Nordlander, P.; Halas, N. J. Hot electrons do the impossible: Plasmon-induced dissociation of $\mathrm{H}_{2}$ on Au. Nano Lett. 2013, 13, 240-247.

[8] Christopher, P.; Xin, H. L.; Marimuthu, A.; Linic, S. Singular characteristics and unique chemical bond activation mechanisms of photocatalytic reactions on plasmonic nanostructures. Nat. Mater. 2012, 11, 1044-1050.

[9] Brongersma, M. L.; Halas, N. J.; Nordlander, P. Plasmoninduced hot carrier science and technology. Nat. Nanotechnol. 2015, 10, 25-34.

[10] Sundaramurthy, A.; Schuck, P. J.; Conley, N. R.; Fromm, D. P.; Kino, G. S.; Moerner, W. E. Toward nanometer-scale optical photolithography: Utilizing the near-field of bowtie optical nanoantennas. Nano Lett. 2006, 6, 355-360.
[11] Ueno, K.; Juodkazis, S.; Shibuya, T.; Yokota, Y.; Mizeikis, V.; Sasaki, K.; Misawa, H. Nanoparticle plasmon-assisted two-photon polymerization induced by incoherent excitation source. J. Am. Chem. Soc. 2008, 130, 6928-6929.

[12] Ueno, K.; Takabatake, S.; Nishijima, Y.; Mizeikis, V.; Yokota, Y.; Misawa, H. Nanogap-assisted surface plasmon nanolithography. J. Phys. Chem. Lett. 2010, 1, 657-662.

[13] Geldhauser, T.; Kolloch, A.; Murazawa, N.; Ueno, K.; Boneberg, J.; Leiderer, P.; Scheer, E.; Misawa, H. Quantitative measurement of the near-field enhancement of nanostructures by two-photon polymerization. Langmuir 2012, 28, 9041-9046.

[14] Gruber, C.; Hirzer, A.; Schmidt, V.; Trügler, A.; Hohenester, U.; Ditlbacher, H.; Hohenau, A.; Krenn, J. R. Imaging nanowire plasmon modes with two-photon polymerization. Appl. Phys. Lett. 2015, 106, 081101.

[15] Kim, N. H.; Meinhart, C. D.; Moskovits, M. Plasmon-mediated reduction of aqueous platinum ions: The competing roles of field enhancement and hot charge carriers. J. Phys. Chem. C 2016, 120, 6750-6755.

[16] Govorov, A. O.; Richardson, H. H. Generating heat with metal nanoparticles. Nano Today 2007, 2, 30-38.

[17] Gonçalves, M.; Trigo, I.; Dias, R.; Costa, M. R. Kinetic modeling of the molecular architecture of cross-linked copolymers synthesized by controlled radical polymerization techniques. Macromol. Symp. 2010, 291-292, 239-250.

[18] Deeb, C.; Ecoffet, C.; Bachelot, R.; Plain, J.; Bouhelier, A.; Soppera, O. Plasmon-based free-radical photopolymerization: Effect of diffusion on nanolithography processes. $\mathrm{J}$. Am. Chem. Soc. 2011, 133, 10535-10542.

[19] Deeb, C.; Zhou, X.; Plain, J.; Wiederrecht, G. P.; Bachelot, R.; Russell, M.; Jain, P. K. Size dependence of the plasmonic near-field measured via single-nanoparticle photoimaging. $J$. Phys. Chem. C 2013, 117, 10669-10676.

[20] Stamplecoskie, K. G.; Pacioni, N. L.; Larson, D.; Scaiano, J. C. Plasmon-mediated photopolymerization maps plasmon fields for silver nanoparticles. J. Am. Chem. Soc. 2011, 133, 9160-9163.

[21] Chen, Y. Q.; Bi, K. X.; Wang, Q. J.; Zheng, M. J.; Liu, Q.; Han, Y. X.; Yang, J. B.; Chang, S. L.; Zhang, G. H.; Duan, H. G. Rapid focused ion beam milling based fabrication of plasmonic nanoparticles and assemblies via "sketch and peel” strategy. ACS Nano 2016, 10, 11228-11236.

[22] Chen, Y. Q.; Xiang, Q.; Li, Z. Q.; Wang, Y. S.; Meng, Y. H.; Duan, H. G. "Sketch and peel" lithography for high-resolution multiscale patterning. Nano Lett. 2016, 16, 3253-3259.

[23] Sakamoto, T.; Kawaura, H.; Baba, T.; Iizuka, T. Direct observation of hot-electron energy distribution in silicon metal-oxide-semiconductor field-effect transistors. Appl. Phys. Lett. 1999, 75, 1113-1115.

[24] Johnson, P. B.; Christy, R. W. Optical constants of the noble metals. Phys. Rev. B 1972, 6, 4370-4379. 